\title{
Factors associated with malnutrition in long-term care versus community dwelling older Irish adults
}

\author{
M. Kelly ${ }^{1}$, H. Purtill ${ }^{2,5}$, M. Grace ${ }^{2}$, S. Leahy ${ }^{2}$, P.W. O'Toole ${ }^{3,4}$, E. O’Herlihy ${ }^{3}$ and \\ E.M. O'Connor ${ }^{1,4,5}$ \\ ${ }^{1}$ Department of Biological Sciences, School of Natural Sciences, University of Limerick, Limerick, ${ }^{2}$ Department of \\ Mathematics and Statistics, University of Limerick, Limerick, ${ }^{3}$ School of Microbiology, University College Cork, Cork, \\ Ireland, ${ }^{4}$ Alimentary Pharmabiotic Centre, Microbiome Institute, University College Cork, Cork, Ireland and \\ ${ }^{5}$ Health Research Institute, University of Limerick, Limerick
}

The prevalence of malnutrition varies widely across elderly population subgroups; it is recognised as being higher in older adults with increasing disabilities, deteriorating health and functional status ${ }^{(1)}$. Since older individuals are living longer and constitute a larger percentage of the global population, it is imperative that malnutrition is detected early to develop strategies for prevention $\&$ management $^{(2)}$. The European Joint Programme Initiative 'Malnutrition in the Elderly' (MaNuEL) was created with the aim of identifying factors associated with malnutrition to develop effective interventions to prevent malnutrition in the elderly in various healthcare settings.

The ELDERMET database comprising information on free living and long-term care older adults was used to determine factors associated with malnutrition, measured at baseline, three and six-month follow-up. Malnutrition was defined as a body mass index (BMI) of $<20 \mathrm{~kg} / \mathrm{m}^{2}$ and/or having a Mini-Nutritional Assessment score of $<17$. Statistically analysis was conducted using SPSS Version $22 \cdot 0$ (IBM Corporation).

Four hundred and forty-six participants were included in the analysis. The baseline analysis identified 78 (17.5\%) participants to be malnourished. Logistic regression analysis found long-term care (adjusted OR $=6 \cdot 35,95 \%$ CI $(3 \cdot 24,12 \cdot 45)$ ) and age (adjusted OR $=$ $1 \cdot 10,95 \%$ CI $(1.05,1 \cdot 15))$ to be significantly associated with malnutrition. Incidence of malnutrition was examined by considering a weight loss of more than $2.25 \mathrm{~kg}$ at the six-month follow-up for those individuals who were not malnourished at baseline and who provided data at six months $(\mathrm{n}=190)$. Unadjusted logistic regression analysis identified age, Functional Independence Measure (FIM), Mini-Mental State Examination (MMSE), Mini-Nutritional Assessment (MNA) and setting (community versus long-term care) as significantly association with incidence malnutrition $(\mathrm{p}<0.05)$. Logistic regression analysis found long-term care to be associated with incident weight loss (adjusted OR $=5 \cdot 35,95 \% \mathrm{CI}(2 \cdot 19,13 \cdot 04)$ ), controlling for age, sex and BMI at baseline.

There are many factors that may contribute to the development of malnutrition in the elderly. Long-term care and age were significantly associated with malnutrition in the ELDERMET cohort. These findings provide evidence that may help identify older adults who are likely to develop malnutrition in the future and thus may contribute to the prevention/early detection of malnutrition.

This research is funded by the Department of Agriculture, Food and the Marine (DAFM) and the Health Research Board (HRB) under the Joint Programming Initiative (JPI) 'Healthy Diet for a Healthy Life' (HDHL) Knowledge Hub on Malnutrition in the Elderly (MaNuEL).

1. Visser M, Volkert D, Corish C et al. (2017) Nutr Bulletin 42, 178-186.

2. Global Health and Aging (2011) [online] World Health Organisation. Available at: http://www.who.int/ageing/publications/global_health.pdf [Accessed 9 Oct. 2017] 